NBER WORKING PAPER SERIES

\title{
CHILD MENTAL HEALTH AND HUMAN CAPITAL ACCUMULATION: THE CASE OF ADHD REVISITED
}

\author{
Jason Fletcher \\ Barbara L. Wolfe \\ Working Paper 13474 \\ http://www.nber.org/papers/w13474 \\ NATIONAL BUREAU OF ECONOMIC RESEARCH \\ 1050 Massachusetts Avenue \\ Cambridge, MA 02138 \\ October 2007
}

This research uses data from Add Health, a program project designed by J. Richard Udry, Peter S. Bearman, and Kathleen Mullan Harris, and funded by a grant P01-HD31921 from the National Institute of Child Health and Human Development, with cooperative funding from 17 other agencies. Special acknowledgment is due Ronald R. Rindfuss and Barbara Entwisle for assistance in the original design. Persons interested in obtaining data files from Add Health should contact Add Health, Carolina Population Center, 123 W. Franklin Street, Chapel Hill, NC 27516-2524 (addhealth@unc.edu). The views expressed herein are those of the author(s) and do not necessarily reflect the views of the National Bureau of Economic Research.

(C) 2007 by Jason Fletcher and Barbara L. Wolfe. All rights reserved. Short sections of text, not to exceed two paragraphs, may be quoted without explicit permission provided that full credit, including $\odot$ notice, is given to the source. 
Child Mental Health and Human Capital Accumulation: The Case of ADHD Revisited Jason Fletcher and Barbara L. Wolfe

NBER Working Paper No. 13474

October 2007, Revised February 2008

JEL No. I1,I2

\begin{abstract}
$\underline{\text { ABSTRACT }}$
Recently, Currie and Stabile (2006) made a significant contribution to our understanding of the influence of ADHD symptoms on a variety of school outcomes including participation in special education, grade repetition and test scores. Their contributions include using a broad sample of children and estimating sibling fixed effects models to control for unobserved family effects. In this paper we look at a sample of older children and confirm and extend many of the JCMS findings in terms of a broader set of measures of human capital and additional specifications.
\end{abstract}

Jason Fletcher

Yale University

School of Public Health

60 College Street, \#303

New Haven, CT 06510

jason.fletcher@yale.edu

Barbara L. Wolfe

1225 Observatory Dr

University of Wisconsin

Madison, WI 53706

and NBER

BWolfe@wisc.edu 


\section{Introduction}

Currie and Stabile (2006) (hereafter, JCMS) made a significant contribution to our understanding of the influence of ADHD symptoms on a variety of school outcomes including participation in special education, grade repetition and test scores. They did so using samples of children ages 4-12 who are then tracked for 6 years, in the United States and Canada. Their contributions include using a broad sample of children, including symptoms rather than only diagnosed cases of ADHD and estimating sibling fixed effects models to control for unobserved family effects. In this paper we extend their findings by looking at a sample of slightly older children and confirm and extend many of the JCMS findings in terms of a broader set of measures of human capital.

Our contribution is to explore the issue of the impact of ADHD on a sample of somewhat older children and examine educational outcomes that occur somewhat later in life. We also explore family effects: that is, does having a sibling with ADHD play a role in human capital accumulation? We first report on the consistency of our results with those of JCMS using both a full sample and then a sibling or fixed effects approach. We then ask if the effect of ADHD on outcomes of older children is as negative as those on younger children. Finally we report on findings relating to family effects; that is, is the human capital accumulation of children who have a sibling with ADHD negatively affected? Following JCMS we also use responses to a set of symptoms of ADHD rather than focusing only on children with ADHD diagnosis.

As noted in JCMS, ADHD is the most common mental illness among young children. Children with ADHD may be hyperactive, inattentive or both. As such, they are more likely to have difficulties in concentrating and carrying out tasks in school, may be disorderly at home and 
in school and may be disruptive to those around them. With the exception of JCMS and recent papers by Fletcher and Wolfe (2007) and Fletcher and Lehrer (2007), most studies of the outcomes of ADHD on children do not control for other factors which may be associated with the both the frequency of ADHD and these poor outcomes, such as living in disadvantaged communities and/or having parents with low levels of human capital. We follow these studies in using family fixed effects in an attempt to control for unobserved factors that might lie behind the prevalence of ADHD and negative outcomes.

We conduct our analysis using the restricted version of the National Longitudinal Study of Adolescent Health (Add Health). Add Health is a school-based, longitudinal study of the health-related behaviors of adolescents and their outcomes in young adulthood. Beginning with an in-school questionnaire administered to a nationally representative sample of students in grades 7 through 12 in 1994-95, the data include follows up with a series of in-home interviews of students approximately one year and then six years later. Additional data are derived from questionnaires filled in by parents, siblings, fellow students, and school administrators. Our measure of ADHD is based on a series of survey questions that are more comprehensive than that used by JCMS; however, they are retrospective in that they are asked of individuals when they are young adults (ages 18-28) and are asked to reflect on their experiences while they were ages 5-12. Compared to the information used by JCMS, our measure of ADHD has the advantage of self reporting (individuals report their own symptoms) and a larger set of questions but the disadvantage of being retrospective. Because the questionnaires differ, the continuous scales, though similar in concept, are not equivalent, in our comparative work with JCMS, we 
focus on the 90\% cut-off indicator of ADHD but also convert the estimated coefficients for the specifications that include the scale scores to be as comparable as possible.

Table 1 provides a comparison of the data set we are using with the U.S. based data set used by JCMS. The first item is a comparison of the ADHD frequency. As noted above, the two data sets use a somewhat different measure, though both are survey based and look at characteristics. The Add Health measure is a retrospective measure filled in by the individual themselves during Wave III when respondents were 18-28 years of age. The survey asks respondents to think back to when they were between 5 and 12 and report how often they performed a set of behaviors (e.g. squirmed in their seat, had difficulty sustaining attention in tasks). ${ }^{1}$ In contrast, the measured used by JCMS was asked of parents of children ages 4-14 and 5 of 26 questions were used to create the hyperactivity scale. Both then refer to approximately the same childhood ages and are based on symptoms rather than only on diagnosis. The first row illustrates the higher mean number of questions on the Add Health scale but both show that the full sample and sibling sample are similar. ${ }^{2}$ The second and third rows report the ADHD measure using a cut-off for significant presence of ADHD symptoms. For both data sets, males are much more likely to be above this threshold than females. Again, the full sample and sibling subsamples are similar. The slightly lower value for the Add Health data is based on our choice to link the measure to clinical significance. The included measure closely mirrors the symptoms

\footnotetext{
${ }^{1}$ One item asked in the retrospective ADHD section of wave III ("You were spiteful or vindictive") is not a DSMIV ADHD symptom and was excluded from analyses; while 1 DSM-IV impulsivity symptom ("Often interrupts or intrudes on others") was not included in the retrospective ADHD section. Thus, our analyses included responses to 9 inattentive and 8 hyperactive/impulsive symptoms. Following Kollins et al. (2005) as well as community based samples (Murphy and Barley 1996), a symptom was considered present if it was experienced "often" or "very often.”

${ }^{2}$ Only the ADHD scale score is statistically different between the full and sibling sample in the Add Health data.
} 
serving as the criteria for diagnosing ADHD in the 2000 Diagnostic and Statistical Manual for Mental Disorders (DSM-IV-TR). ${ }^{3}$

The grade repetition indicator refers to years 1994-2000 for the NLS-Y data and grades 7-12 for Add Health. When we add grade 6 so that the same number of years are in both, the means are quite similar (5.8\% vs. 6.8\%). We chose to only examine this outcome in grades 7-12, however, as we prefer an indicator of grade repetition following the report of symptoms, which are asked for ages 5-12. ${ }^{4}$ Special education indicators are quite similar across data sets and across the full and sibling data sets. ${ }^{5}$ Finally, in terms of family background, we note that family income (in 1994) is remarkably similar across data sets while the mother's education suggests somewhat better educated mothers among the Add-Health population. (This may reflect the oversampling of disadvantaged individuals in the NLS-Y.) The difference in child's age reflects the different sampling strategies of the two samples and also suggests our ability to look at longer term outcomes compared to the NLS-Y data analysis.

\section{$\underline{\text { Results }}$}

We move now to estimation of the relationship of the human capital and ADHD symptoms. The equations take two forms:

(1) $Y_{i}=\alpha+\beta A D H D_{i}+\lambda X_{i}+\varepsilon_{i}$

\footnotetext{
${ }^{3}$ The DSM-IV-TR criteria can be found at: http://www.cdc.gov/ncbddd/adhd/symptom.htm. The Add Health survey contains all items except the impulsivity question, "Often interrupts or intrudes on others."

${ }^{4}$ Our results do not significantly change if we use grades 6-12 rather than 7-12.

${ }^{5}$ The NLS-Y measure includes parent reports for the year 2000 when children were approximately 8-16 years old. The Add Health measure includes parent reports for the year 1994/95 when the children were approximately 12-18 years old.
} 
where $Y_{i}$ is one of the human capital outcomes for child $i, A D H D$ is an indicator of a child's ADHD symptoms over ages 5-12, $X$ is a set of additional covariates and $\beta$ and $\lambda$ are coefficients to be estimated, and

(2) $Y_{i f}=\alpha+\beta A D H D_{i f}+\lambda Z_{i f}+\mu_{f}+\varepsilon_{i}$

where $\mathrm{Z}$ is a subset of covariates $\mathrm{X}$ that differ between siblings, and $f$ indexes families or siblings.

Replication Results: Our initial set of estimates are quite similar to JCMS and are reported in Table $2 .{ }^{6}$ To show comparability between our results and JCMS, we initially focus on grade repetition and special education. The first results shown in Table 2 are those using dummy variable indicators for ADHD in the NLSY and for Add Health. The Add Health measure reflects the clinically relevant cutoffs for ADHD described above and the NLSY measure indicates whether the child is in the upper decile of the hyperactivity scale. ${ }^{7}$ These results in rows 1 and 2 that use dummy variables are very similar with coefficients of .06 versus .07 for grade repetition and .08 and .12 for special education. The next two rows report results using the full scale. Because the scales differ in the two samples, we multiply the coefficients by the standard deviation of the scale scores (8.89 in Add Health and 3.81 in the NLSY) to obtain adjusted or standardized coefficients. The resulting estimates are nearly identical for the two samples. The standardized coefficients for grade repetition are .018 for Add Health and .015 for NLSY; for special education they are .031 and .028 respectively. All four adjusted coefficients are statistically significant at the 95 percent level. Both sets of results thus provide evidence that

\footnotetext{
${ }^{6}$ Full results for all tables are presented in the appendix.
} 
the mental health symptoms of ADHD are tied to statistically significant increased probabilities of grade repetition and of being placed in special education. ${ }^{8}$ We consider the results using Add Health to closely replicate those of NLSY and hence should be viewed as comparable to the JCMS results. On this basis we proceed to extend the JCMS results by considering longer term educational outcomes.

Extended Results: Because the children in Add Health are older, we are able to analyze longer run human capital outcomes. We do this below first for those within secondary schools and then move on to longer term measures of human capital using the same sample and same approach - OLS followed by family fixed effects.

We begin with grade point average, computed as the average letter-grade in mathematics, English, history, and science classes during wave $1 .{ }^{9}$ Here we find that the OLS results suggest on average a quarter point lower GPA for those with ADHD symptoms; once family fixed effects are taken into account this is reduced somewhat-to -.15 and is no longer statistically significant at the $95 \%$ level. This change may be due solely to the addition of unobserved family factors or may also tie to the smaller sample size. Nevertheless, taken together we view these results as an indication of a negative influence of ADHD on grades.

\footnotetext{
${ }^{7}$ As mentioned above, the Add Health scale is based on DSM-IV criteria whereas the NLSY scale is somewhat ad hoc and is based on five hyperactivity questions available in the 26-question Behavior Problems Index.

${ }^{8} \mathrm{JCMS}$ find that using their ADHD cutoff, boys are more likely to be placed in special education and that negative effects on test scores are confined to boys. Our results are roughly consistent with those of JCMS. We find that boys with ADHD symptoms are more likely to be placed in special education, but the difference is not statistically significant $(4 \%$, p-value <.14). We also find suggestive evidence that boys with ADHD are more likely to repeat a grade $(2.6 \%$, p-value $<.11)$. Full results are presented in the appendix.

${ }_{9}^{9}$ In cases where students were not enrolled in a particular subject (e.g. history), their grades for their enrolled subjects were used.
} 
Turning to our other within secondary school indicators, Suspended, Expelled and Dropout, we find that in every case of these measures of human capital at older ages, the full sample results suggest a link between ADHD symptoms and higher probabilities of these outcomes but the FE estimates are smaller than the full sample estimates. Only in the case of suspensions is there any finding of a link between ADHD and this outcome once fixed effects are estimated. In the case of suspensions, the higher probability estimated in both models is approximately 0.15 . Finally when we look at longer term measures of years of education and probability of attending college, our FE estimates suggest there is not a significant influence of ADHD on either of these measures of length of schooling once unobserved family effects are taken into account. (Our full sample results are consistent with the existing literature which finds a negative and sizeable negative influence of ADHD on years of schooling, the probability of not completing high school and the lower probability of attending college).

At first glance then, it appears that the influence of ADHD on human capital outcomes are short-term in nature, once unobserved family effects are taken into account. The results appear stronger in the sense that the results for shorter term outcomes are generally consistent with those of JCMS.

The results for longer term educational outcomes seem somewhat counter-intuitive. We would expect that the influence of ADHD might indeed reduce human capital, and if not cumulative, would have a long run influence. To explore this, we developed another hypothesis: that ADHD has negative influences on not just the child with the symptoms, but also on others such as family members and in particular, on siblings. This could occur as families over longer periods of time attempt to compensate the child having ADHD, which would reduce 
investments, including time, with the other children, and lower measured differences between siblings. Or there could be a direct negative influence of living with a disruptive sibling who has ADHD. Thus we estimate a third equation to capture the idea that having a sibling with ADHD may have a negative influence on a child's human capital accumulation:

(3) $Y_{i}=\alpha+\beta A D H D_{i}+\delta A D H D_{j}+\lambda X_{i}+\varepsilon_{i}$

where $A D H D_{j}$ indicates the presence of another child in this child's family who has ADHD.

The result presented in Table 4 are consistent with our hypothesis: having a sibling with ADHD reduces the human capital of the child with ADHD and the longer run measures of human capital for additional children in the household. These results then suggest that other family fixed effects results underestimate the human capital consequences of ADHD for they only have analyzed the individual directly affected. Here we find that siblings also suffer a long term reduction in human capital. Since these estimates which are based on equation (3) do not contain family fixed effects ${ }^{10}$ they are subject to omitted variables bias. To test the sensitivity of these results, we first estimate a specification with an extended number of family-level characteristics and subsequently estimate a random effects specification. We find the sibling spillover results presented in Table 4 to be robust (see appendix table 10A). ${ }^{11}$

\section{Conclusion}

\footnotetext{
${ }^{10}$ In this context, family fixed effects are collinear with the inclusion of dummy variables indicating each siblings' ADHD status and we would only be able to identify the sibling ADHD spillover effects off of families with more than two siblings in the data who are discordant in ADHD status (less than 40 individuals).

${ }^{11}$ Including the community unemployment rate, the community proportion in poverty, maternal smoking status, father's education level, parent's general health status, parent's age, reports of parents' alcoholism status, receipt of AFDC or Food Stamps, and parental reports of education expectations decreases the coefficient on sibling spillovers
} 
In this paper, we corroborate the short-term educational consequences of ADHD shown by JCMS and extend the examination to longer-term educational outcomes of children with ADHD symptoms. Like the results by JCMS for the children in the NLS-Y, we find evidence that children in the Add Health dataset who have ADHD symptoms are more likely to repeat a grade and receive special education services. We then show that standard OLS results imply that children with ADHD face longer-term educational disadvantages, including lower grade point averages, increases in suspension and expulsions, and fewer completed years of schooling. However, we find that nearly all of these results are not robust to the inclusion of family fixed effects, suggesting that short term consequences of educational outcomes do not lead to longer term educational consequences in a straightforward manner.

Why might we expect to find short term but not long term consequences of childhood ADHD symptoms on educational outcomes? We conjecture that over a longer time period, either parents are more able to compensate children with ADHD, which could lead to smaller withinsibling differences in educational outcomes or having a disruptive sibling with ADHD reduces the human capital of other children in the family. Building on this conjecture, we extend the literature by adding measures of ADHD symptoms of siblings to predict own-education outcomes and find evidence that having a sibling with ADHD is harmful to education outcomes. This suggests that the consequences of ADHD extend beyond those found by Currie and Stabile and strengthen their call for additional school resources to focus on those with ADHD symptoms.

by approximately $15 \%$ across outcomes. See appendix Table $10 \mathrm{~A}$ for results. 


\section{References}

American Psychiatric Association: Diagnostic and Statistical Manual of Mental Disorders, Fourth Edition, Text Revision. Washington, DC, American Psychiatric Association, 2000.

Currie, Janet and Mark Stabile. "Child Mental Health and Human Capital Accumulation: The Case of ADHD.” 2006. Journal of Health Economics, 25: 6. 1094-1118.

Fletcher, Jason M. and Steven F. Lehrer. "Using the 'Genetic Lottery' within Families to Examine the Effects of Health on Education.” Working Paper 2007, Presented at the NBER 2007 Summer Institute

Fletcher, Jason M. and Barbara L. Wolfe. "Long Term Consequences of Childhood ADHD on Criminal Activities.” Working Paper 2007, Presented at the 2007 Population Association of America Annual Meeting

Kollins, Scott H, F. Joseph McClernon, and Bernard Fuemmeler. "Association Between Smoking and Attention-Deficit/Hyperactivity Disorder Symptoms in a Population-Based Sample of Young Adults.” Archives of General Psychiatry, 2005; 62:1142-1147

Murphy K, and R. Barkley "Prevalence of DSM-IV symptoms of ADHD in adult licensed drivers: implications for clinical diagnosis.” Journal of Attention Disorders 1996; 1: 47-161. 


\section{Tables}

\begin{tabular}{|c|c|c|c|c|}
\hline & $\begin{array}{l}\text { Add-Health } \\
\text { Full Sample }\end{array}$ & $\begin{array}{c}\text { Add-Health } \\
\text { Sibling Sample }\end{array}$ & $\begin{array}{c}\text { NLS-Y } \\
\text { Full Sample }\end{array}$ & $\begin{array}{c}\text { NLS-Y } \\
\text { Sibling Sample }\end{array}$ \\
\hline $\begin{array}{l}\text { Scale of Own or parent } \\
\text { reported ADHD score }\end{array}$ & $\begin{array}{c}13.2 \\
(8.90)\end{array}$ & $\begin{array}{l}13.53 \\
(8.89)\end{array}$ & $\begin{array}{c}5.93 \\
(3.79)\end{array}$ & $\begin{array}{c}5.79 \\
(3.81)\end{array}$ \\
\hline ADHD Cutoff (Males) & 0.107 & 0.112 & 0.123 & 0.122 \\
\hline ADHD Cutoff (Females) & 0.063 & 0.061 & 0.075 & 0.073 \\
\hline Grade repetition & 0.057 & 0.056 & .075 & .072 \\
\hline Special education & 0.087 & 0.083 & .071 & .078 \\
\hline Mother high school or more & .85 & .85 & .75 & .75 \\
\hline Family income (94\$) & 45066 & 44603 & 41483 & 42185 \\
\hline Child Age in $1994 / 1995$ & $\begin{array}{l}17.12 \\
(1.73)\end{array}$ & $\begin{array}{l}17.16 \\
(1.69)\end{array}$ & $\begin{array}{c}8.27 \\
(2.30)\end{array}$ & $\begin{array}{c}8.17 \\
(2.26)\end{array}$ \\
\hline Number of Observations $^{12}$ & $\sim 14,000$ & $\sim 3,000$ & 3,969 & 2,406 \\
\hline
\end{tabular}

Notes: Add Health = National Longitudinal Study of Adolescent Health (1994-2001).

NLS-Y = National Longitudinal Survey of Youth (1990-1994, 1998-2000).

\footnotetext{
${ }^{12}$ A table of summary statistics provided in the appendix shows the number of observations available for each variable in the Add Health data.
} 


\begin{tabular}{|l|l|l|}
\hline \multicolumn{3}{|l|}{$\begin{array}{l}\text { Table 2: Association between ADHD and Grade Repetition and Being in Special Education: A } \\
\text { Comparison of full sample and family FE Results across Two Samples } \dagger\end{array}$} \\
\hline & Grade repetition & \\
\hline & .33 & Special Education \\
\hline Add Health FE, ADHD as dummy variable & $.060^{*}$ & .077 \\
\hline $\begin{array}{l}\text { NLSY FE, dummy variable for ADHD score }> \\
90^{\text {th }} \text { percentile }\end{array}$ & $.070^{* *}$ & $.121^{* *}$ \\
\hline Add Health-full sample (scale score) & $0.018^{* *}$ & $0.036^{* *}$ \\
\hline NLSY-full sample (scale score) & $0.015^{* *}$ & $0.031^{* *}$ \\
\hline Add Health FE (scale score) & $0.018^{*}$ & $0.028^{*}$ \\
\hline NLSY FE (scale score) & $0.019^{*}$ & $0.034^{*}$ \\
\hline
\end{tabular}

Note: ** Statistically significant at $95 \%$ level * statistically significant at $90 \%$ level.

$\dagger$ Equations also include sex, race, birth order, indicator of child's health status, family income, family size, marital status of parents, mother's education and child's age.

Coefficients in rows 3-6 represent the estimated coefficients of the scale score

multiplied by the standard deviation of the scale scores (8.89 in Add Health and 3.81 in

the NLSY) for comparability between scales. Full results for the Add Health

specifications (Rows 1, 3, and 5) can be found in Tables 2A and 3A in the appendix.

Row 4 results found in Table 3 of JCMS. Rows 2 and 6 results found in Table 4 of

JCMS.

\footnotetext{
${ }^{13}$ Grade repetition results are nearly identical if we use a measure of grade repetition for grades 6-12 and are available upon request.
} 


\begin{tabular}{|c|c|c|}
\hline & Full Sample $(\sim 14,000)$ & Sibling FE ( 2900) \\
\hline GPA & $-.25 * *$ & -.15 \\
\hline Suspended & $.16^{* *}$ & $.13^{* *}$ \\
\hline Expelled & $.03 * *$ & .010 \\
\hline Drop out & $.10^{* *}$ & -.013 \\
\hline Years of education & $-.52 * *$ & .205 \\
\hline Attend College & $-.08 * *$ & .003 \\
\hline
\end{tabular}

Notes: ** Statistically significant at $95 \%$ level * statistically significant at $90 \%$ level. $\dagger$ Equations also include sex, race, birth order, indicator of child's health status, family income, family size, marital status of parents, mother's education and child's age in full model. For the outcome "attend college" we drop individuals who do not complete high school from the sample. Sample sizes vary for the full sample between 14,068 and 12,511 depending on the outcome. For the family sample, the sample size varies between 3.019 and 2,662. Results using the ADHD scale rather than a binary indicator as well as using the $90 \%$ cutoff of the ADHD scale (following JCMS) are quite similar and are available upon request. 


\begin{tabular}{|l|c|c|c|}
\hline \multicolumn{2}{|l|}{ Table 4: Estimates adding Sibling has ADHD to Family Sample† } \\
\hline & Own ADHD results & \multicolumn{2}{l|}{ Sibling with ADHD results } \\
\hline & & own ADHD & Sib with ADHD \\
\hline Years of School & $-0.223^{*}$ & -0.205 & $-0.346^{* *}$ \\
\hline Drop-out & $0.066^{* *}$ & $0.064^{* *}$ & $0.045^{* *}$ \\
\hline Attend College & $-0.061^{*}$ & $-0.055^{*}$ & $-0.092^{* *}$ \\
\hline GPA & $-0.188^{* *}$ & $-0.185^{* *}$ & -0.057 \\
\hline Expelled & 0.014 & 0.013 & 0.008 \\
\hline Suspended & $0.174^{* *}$ & $0.171^{* *}$ & $0.066^{* *}$ \\
\hline Grade repetition & $0.050^{* *}$ & $0.050^{* *}$ & -0.013 \\
\hline Special Education & $0.059^{* *}$ & $0.060^{* *}$ & -0.008 \\
\hline
\end{tabular}

Notes: ** Statistically significant at $95 \%$ level * statistically significant at $90 \%$ level.

$\dagger$ Equations also include sex, race, birth order, indicator of child's health status, family income, family size, marital status of parents, mother's education and child's age in full model. 


\section{Appendix Tables}

Table $1 \mathrm{~A}$

Complete Summary Statistics for Full and Sibling Samples in Add Health

\begin{tabular}{|lrrr|rrr}
\hline \multicolumn{1}{c}{ Variable } & Obs & \multicolumn{1}{c|}{ Mean } & Std Dev & \multicolumn{1}{c}{ Obs } & Mean & Std Dev \\
\hline & Full & & & \multicolumn{1}{c}{ Sibling } & & \\
Sample & & & Sample & & \\
ADHD Cutoff & 14306 & 0.08 & 0.28 & 2907 & 0.09 & 0.28 \\
ADHD Scale & 14306 & 13.22 & 8.90 & 2907 & 13.65 & 8.95 \\
Sibling with ADHD & & & & 2907 & 0.09 & 0.29 \\
Repeat Grade (7th-12th) & 14297 & 0.06 & 0.23 & 2904 & 0.05 & 0.22 \\
Suspended (7th-12th) & 13803 & 0.24 & 0.43 & 2804 & 0.25 & 0.43 \\
Special Education & 12389 & 0.09 & 0.28 & 2570 & 0.08 & 0.27 \\
Expelled (7th-12th) & 14202 & 0.03 & 0.18 & 2886 & 0.03 & 0.17 \\
Grade Point Average & 13907 & 2.78 & 0.77 & 2836 & 2.78 & 0.77 \\
Years of Schooling & 14307 & 13.22 & 1.97 & 2907 & 13.16 & 1.99 \\
Dropped Out & 14125 & 0.11 & 0.32 & 2865 & 0.12 & 0.32 \\
College Enrollment & 12725 & 0.67 & 0.47 & 2572 & 0.64 & 0.48 \\
Age, Wave I & 14312 & 17.12 & 1.73 & 2909 & 17.16 & 1.69 \\
Male & 14320 & 0.47 & 0.50 & 2910 & 0.48 & 0.50 \\
Black & 14320 & 0.22 & 0.41 & 2910 & 0.22 & 0.42 \\
Hispanic & 14320 & 0.16 & 0.36 & 2910 & 0.13 & 0.34 \\
Other race & 14320 & 0.08 & 0.28 & 2910 & 0.08 & 0.28 \\
General Health Status & & & & & & \\
(1=poor, 5=excellent) & 14311 & 3.88 & 0.91 & 2909 & 3.88 & 0.91 \\
Birth Order & 14304 & 1.85 & 1.20 & 2906 & 2.05 & 1.29 \\
Parent Age & 14320 & 42.05 & 6.16 & 2910 & 41.64 & 5.88 \\
Maternal Education (Yrs.) & 14320 & 13.21 & 2.28 & 2910 & 13.12 & 2.24 \\
Family Income (\$10,000s) & 14320 & 46.07 & 40.55 & 2910 & 45.00 & 41.77 \\
Married Household & 14320 & 0.62 & 0.48 & 2910 & 0.63 & 0.48 \\
Number of Siblings & 14110 & 1.62 & 1.45 & 2861 & 2.02 & 1.56 \\
\hline
\end{tabular}


Table 2A

Association between ADHD Symptoms and Grade Repetition Results using ADHD Dummy Variable

\begin{tabular}{|c|c|c|c|c|c|}
\hline Outcome & $\begin{array}{l}\text { Repeat } \\
\text { Grade }\end{array}$ & $\begin{array}{l}\text { Repeat } \\
\text { Grade }\end{array}$ & $\begin{array}{l}\text { Repeat } \\
\text { Grade }\end{array}$ & $\begin{array}{l}\text { Repeat } \\
\text { Grade }\end{array}$ & $\begin{array}{l}\text { Repeat } \\
\text { Grade }\end{array}$ \\
\hline Sample & Full & Full & Family & Family & Family \\
\hline Fixed Effects? & None & None & None & None & Family \\
\hline \multirow{2}{*}{ ADHD Status } & 0.045 & 0.030 & 0.050 & 0.050 & 0.059 \\
\hline & $(0.000)^{\star \star}$ & $(0.016)^{\star}$ & $(0.011)^{\star}$ & $(0.009)^{\star \star}$ & $(0.034)^{\star}$ \\
\hline \multirow[t]{2}{*}{ Male } & 0.037 & 0.035 & 0.030 & 0.031 & 0.040 \\
\hline & $(0.000)^{\star \star}$ & $(0.000)^{\star *}$ & $(0.000)^{\star \star}$ & $(0.000)^{\star \star}$ & $(0.019)^{\star \star}$ \\
\hline \multirow[t]{2}{*}{ Age } & 0.020 & 0.020 & 0.021 & 0.021 & 0.019 \\
\hline & $(0.000)^{\star \star}$ & $(0.000)^{\star \star}$ & $(0.000)^{\star \star}$ & $(0.000)^{\star \star}$ & $(0.008)^{\star \star}$ \\
\hline \multirow[t]{2}{*}{ Black } & 0.022 & 0.022 & 0.012 & 0.011 & \\
\hline & $(0.021)^{\star}$ & $(0.019)^{\star}$ & $(0.307)$ & $(0.324)$ & \\
\hline \multirow[t]{2}{*}{ Hispanic } & 0.018 & 0.019 & 0.025 & 0.025 & \\
\hline & $(0.069)+$ & $(0.068)+$ & $(0.116)$ & $(0.116)$ & \\
\hline \multirow[t]{2}{*}{ Other Race } & -0.006 & -0.006 & -0.002 & -0.002 & \\
\hline & (0.614) & $(0.624)$ & $(0.922)$ & $(0.886)$ & \\
\hline \multirow[t]{2}{*}{ General Health Status } & -0.018 & -0.018 & -0.016 & -0.016 & -0.009 \\
\hline & $(0.000)^{\star \star}$ & $(0.000)^{\star \star}$ & $(0.001)^{\star \star}$ & $(0.001)^{\star \star}$ & $(0.012)$ \\
\hline \multirow[t]{2}{*}{ Birth Order } & -0.001 & -0.001 & -0.002 & -0.002 & -0.012 \\
\hline & $(0.608)$ & $(0.610)$ & $(0.677)$ & $(0.687)$ & $(0.016)$ \\
\hline \multirow[t]{2}{*}{ Parental Age } & -0.001 & -0.001 & -0.002 & -0.002 & \\
\hline & $(0.010)^{\star \star}$ & $(0.010)^{\star \star}$ & $(0.016)^{\star}$ & $(0.016)^{\star}$ & \\
\hline \multirow[t]{2}{*}{ Maternal Education } & -0.005 & -0.005 & -0.001 & -0.001 & \\
\hline & $(0.001)^{\star \star}$ & $(0.001)^{\star \star}$ & $(0.422)$ & $(0.443)$ & \\
\hline \multirow[t]{2}{*}{ Family Income } & -0.000 & -0.000 & -0.000 & -0.000 & \\
\hline & $(0.006)^{\star \star}$ & $(0.006)^{\star \star}$ & $(0.482)$ & $(0.485)$ & \\
\hline \multirow[t]{2}{*}{ Married Household } & -0.027 & -0.027 & -0.031 & -0.032 & \\
\hline & $(0.000)^{\star \star}$ & $(0.000)^{\star \star}$ & $(0.003)^{\star \star}$ & $(0.003)^{\star \star}$ & \\
\hline \multirow[t]{2}{*}{ Number Siblings } & 0.004 & 0.004 & 0.004 & 0.004 & \\
\hline & $(0.104)$ & $(0.104)$ & $(0.403)$ & $(0.405)$ & \\
\hline \multirow[t]{2}{*}{ Male X Adhd } & & 0.026 & & & \\
\hline & & $(0.104)$ & & & \\
\hline \multirow[t]{2}{*}{ Sibling ADHD } & & & & -0.013 & \\
\hline & & & & $(0.308)$ & \\
\hline \multirow[t]{2}{*}{ Constant } & -0.122 & -0.120 & -0.155 & -0.154 & -0.227 \\
\hline & $(0.003)^{\star \star}$ & $(0.003)^{\star \star}$ & $(0.003)^{\star \star}$ & $(0.003)^{\star \star}$ & $(0.153)$ \\
\hline Observations & 14056 & 14056 & 3015 & 3015 & 3015 \\
\hline R-squared & 0.053 & 0.053 & 0.048 & 0.048 & 0.05 \\
\hline Number of Families & & & & & 1682 \\
\hline
\end{tabular}

Notes: ** Statistically significant at $99 \%$ level * statistically significant at $95 \%$ level, + statistically significant at the $90 \%$ level. All results use robust standard errors clustered at the school or family level. 
Table 2A (continued)

Association between ADHD Symptoms and Grade Repetition

Results using ADHD Scale

\begin{tabular}{|c|c|c|c|c|c|}
\hline Outcome & $\begin{array}{l}\text { Repeat } \\
\text { Grade }\end{array}$ & $\begin{array}{l}\text { Repeat } \\
\text { Grade }\end{array}$ & $\begin{array}{l}\text { Repeat } \\
\text { Grade }\end{array}$ & $\begin{array}{c}\text { Repeat } \\
\text { Grade }\end{array}$ & $\begin{array}{c}\text { Repeat } \\
\text { Grade }\end{array}$ \\
\hline Sample & Full & Full & Family & Family & Family \\
\hline Fixed Effects? & None & None & None & None & Family \\
\hline \multirow[t]{2}{*}{ ADHD Scale } & 0.002 & 0.002 & 0.003 & 0.003 & $0.002^{*}$ \\
\hline & $(0.000)^{\star \star}$ & $(0.000)^{\star \star}$ & $(0.000)^{* \star}$ & $(0.000)^{\star \star}$ & $(0.001)$ \\
\hline \multirow[t]{2}{*}{ Male } & 0.032 & 0.031 & 0.024 & 0.024 & $0.036^{*}$ \\
\hline & $(0.000)^{\star \star}$ & $(0.000)^{\star \star}$ & $(0.005)^{\star \star}$ & $(0.004)^{\star \star}$ & $(0.020)$ \\
\hline \multirow[t]{2}{*}{ Age } & 0.020 & 0.020 & 0.022 & 0.022 & $0.020^{\star \star \star}$ \\
\hline & $(0.000)^{\star \star}$ & $(0.000)^{\star \star}$ & $(0.000)^{\star *}$ & $(0.000)^{\star \star}$ & $(0.008)$ \\
\hline \multirow[t]{2}{*}{ Black } & 0.024 & 0.024 & 0.015 & 0.014 & \\
\hline & $(0.012)^{\star}$ & $(0.011)^{\star}$ & $(0.189)$ & $(0.203)$ & \\
\hline \multirow[t]{2}{*}{ Hispanic } & 0.021 & 0.021 & 0.027 & 0.027 & \\
\hline & $(0.042)^{\star}$ & $(0.041)^{\star}$ & $(0.085)+$ & $(0.084)+$ & \\
\hline \multirow[t]{2}{*}{ Other Race } & -0.005 & -0.004 & 0.001 & -0.000 & \\
\hline & $(0.703)$ & $(0.724)$ & $(0.966)$ & $(0.987)$ & \\
\hline \multirow[t]{2}{*}{ General Health Status } & -0.017 & -0.017 & -0.014 & -0.014 & -0.009 \\
\hline & $(0.000)^{\star \star}$ & $(0.000)^{\star \star}$ & $(0.004)^{\star \star}$ & $(0.004)^{\star \star}$ & $(0.012)$ \\
\hline \multirow[t]{2}{*}{ Birth Order } & -0.002 & -0.002 & -0.002 & -0.002 & -0.012 \\
\hline & $(0.458)$ & $(0.453)$ & $(0.636)$ & $(0.648)$ & $(0.017)$ \\
\hline \multirow[t]{2}{*}{ Parental Age } & -0.001 & -0.001 & -0.002 & -0.002 & \\
\hline & $(0.014)^{\star}$ & $(0.014)^{\star}$ & $(0.015)^{\star}$ & $(0.014)^{\star}$ & \\
\hline \multirow[t]{2}{*}{ Maternal Education } & -0.005 & -0.005 & -0.001 & -0.001 & \\
\hline & $(0.001)^{\star \star}$ & $(0.001)^{\star *}$ & $(0.625)$ & $(0.664)$ & \\
\hline \multirow[t]{2}{*}{ Family Income } & -0.000 & -0.000 & -0.000 & -0.000 & \\
\hline & $(0.009)^{\star \star}$ & $(0.009)^{\star \star}$ & $(0.585)$ & $(0.592)$ & \\
\hline \multirow[t]{2}{*}{ Married Household } & -0.026 & -0.026 & -0.031 & -0.031 & \\
\hline & $(0.000)^{\star \star}$ & $(0.000)^{\star \star}$ & $(0.004)^{\star \star}$ & $(0.003)^{\star \star}$ & \\
\hline \multirow[t]{2}{*}{ Number Siblings } & 0.004 & 0.004 & 0.004 & 0.004 & \\
\hline & $(0.082)+$ & $(0.081)+$ & $(0.362)$ & $(0.364)$ & \\
\hline \multirow[t]{2}{*}{ Male X Adhd } & & 0.019 & & & \\
\hline & & $(0.148)$ & & & \\
\hline \multirow[t]{2}{*}{ Sibling ADHD } & & & & -0.018 & \\
\hline & & & & $(0.168)$ & \\
\hline Observations & 14056 & 14056 & 3015 & 3015 & 3015 \\
\hline R-squared & 0.056 & 0.056 & 0.054 & 0.054 & 0.05 \\
\hline Number of Families & & & & & 1682 \\
\hline
\end{tabular}

Notes: ** Statistically significant at 99\% level * statistically significant at $95 \%$ level, +

statistically significant at the $90 \%$ level. All results use robust standard errors clustered at the school or family level. Results for the constant are omitted. Results in Table 2 in the text are calculated by multiplying the ADHD scale coefficient in Table 2A by the standard deviation of the scale (8.89 in Add Health and 3.81 in NLS-Y) 
Table 3A

Association between ADHD Symptoms and Special Education Placement Results using ADHD Dummy Variable

\begin{tabular}{|c|c|c|c|c|c|}
\hline Outcome & $\begin{array}{c}\text { Special } \\
\text { Ed }\end{array}$ & $\begin{array}{c}\text { Special } \\
\text { Ed }\end{array}$ & $\begin{array}{c}\text { Special } \\
\text { Ed }\end{array}$ & $\begin{array}{c}\text { Special } \\
\text { Ed }\end{array}$ & $\begin{array}{c}\text { Special } \\
\text { Ed }\end{array}$ \\
\hline Sample & Full & Full & Family & Family & Family \\
\hline Fixed Effects? & None & None & None & None & Family \\
\hline \multirow[t]{2}{*}{ ADHD Status } & 0.084 & 0.060 & 0.059 & 0.060 & 0.077 \\
\hline & $(0.000)^{\star \star}$ & $(0.001)^{\star \star}$ & $(0.016)^{\star}$ & $(0.014)^{\star}$ & $(0.050)$ \\
\hline \multirow[t]{2}{*}{ Male } & 0.045 & 0.042 & 0.042 & 0.042 & 0.056 \\
\hline & $(0.000)^{\star \star}$ & $(0.000)^{\star \star}$ & $(0.000)^{\star \star}$ & $(0.000)^{\star \star}$ & $(0.024)^{\star \star}$ \\
\hline \multirow[t]{2}{*}{ Age } & 0.002 & 0.002 & 0.001 & 0.001 & 0.006 \\
\hline & $(0.522)$ & $(0.526)$ & $(0.772)$ & $(0.773)$ & $(0.008)$ \\
\hline \multirow[t]{2}{*}{ Black } & -0.014 & -0.013 & -0.034 & -0.034 & \\
\hline & $(0.201)$ & $(0.209)$ & $(0.018)^{\star}$ & $(0.017)^{\star}$ & \\
\hline \multirow[t]{2}{*}{ Hispanic } & -0.035 & -0.034 & -0.031 & -0.031 & \\
\hline & $(0.001)^{\star \star}$ & $(0.001)^{\star \star}$ & $(0.126)$ & $(0.126)$ & \\
\hline \multirow[t]{2}{*}{ Other Race } & -0.042 & -0.042 & -0.084 & -0.085 & \\
\hline & $(0.004)^{\star \star}$ & $(0.004)^{\star \star}$ & $(0.000)^{\star *}$ & $(0.000)^{\star \star}$ & \\
\hline \multirow[t]{2}{*}{ General Health Status } & -0.016 & -0.016 & -0.018 & -0.018 & -0.016 \\
\hline & $(0.000)^{\star \star}$ & $(0.000)^{\star \star}$ & $(0.006)^{\star \star}$ & $(0.006)^{\star \star}$ & $(0.013)$ \\
\hline \multirow[t]{2}{*}{ Birth Order } & 0.005 & 0.005 & 0.007 & 0.007 & 0.015 \\
\hline & $(0.171)$ & $(0.170)$ & $(0.271)$ & $(0.268)$ & $(0.015)$ \\
\hline \multirow[t]{2}{*}{ Parental Age } & -0.000 & -0.000 & -0.001 & -0.001 & \\
\hline & $(0.316)$ & $(0.314)$ & $(0.280)$ & $(0.277)$ & \\
\hline \multirow[t]{2}{*}{ Maternal Education } & -0.006 & -0.006 & -0.003 & -0.003 & \\
\hline & $(0.001)^{\star \star}$ & $(0.001)^{\star \star}$ & $(0.284)$ & $(0.288)$ & \\
\hline \multirow[t]{2}{*}{ Family Income } & -0.000 & -0.000 & -0.000 & -0.000 & \\
\hline & $(0.174)$ & $(0.182)$ & $(0.313)$ & $(0.314)$ & \\
\hline \multirow[t]{2}{*}{ Married Household } & -0.027 & -0.027 & -0.021 & -0.021 & \\
\hline & $(0.000)^{\star *}$ & $(0.000)^{\star \star}$ & $(0.135)$ & $(0.132)$ & \\
\hline \multirow[t]{2}{*}{ Number Siblings } & -0.004 & -0.004 & -0.003 & -0.003 & \\
\hline & $(0.264)$ & $(0.267)$ & $(0.578)$ & $(0.577)$ & \\
\hline \multirow[t]{2}{*}{ Male X Adhd } & & 0.039 & & & \\
\hline & & $(0.133)$ & & & \\
\hline \multirow[t]{2}{*}{ Sibling ADHD } & & & & -0.008 & \\
\hline & & & & $(0.654)$ & \\
\hline \multirow[t]{2}{*}{ Constant } & 0.223 & 0.225 & 0.219 & 0.219 & -0.023 \\
\hline & $(0.000)^{\star \star}$ & $(0.000)^{\star *}$ & $(0.004)^{\star \star}$ & $(0.004)^{\star \star}$ & $(0.159)$ \\
\hline Observations & 12172 & 12172 & 2668 & 2668 & 2668 \\
\hline R-squared & 0.026 & 0.026 & 0.024 & 0.024 & 0.03 \\
\hline Number of Families & & & & & 1526 \\
\hline
\end{tabular}

Notes: ** Statistically significant at $99 \%$ level * statistically significant at $95 \%$ level, + statistically significant at the $90 \%$ level. All results use robust standard errors clustered at the school or family level. 
Table 3A (continued)

Association between ADHD Symptoms and Special Education Placement

Results using ADHD Scale

\begin{tabular}{|c|c|c|c|c|c|}
\hline Outcome & Special Ed & Special Ed & Special Ed & Special Ed & Special Ed \\
\hline Sample & Full & Full & Family & Family & Family \\
\hline Fixed Effects? & None & None & None & None & Family \\
\hline \multirow[t]{2}{*}{ ADHD Scale } & 0.004 & 0.004 & 0.003 & 0.003 & $0.003^{*}$ \\
\hline & $(0.000)^{\star \star}$ & $(0.000)^{\star \star}$ & $(0.000)^{\star \star}$ & $(0.000)^{\star \star}$ & $(0.001)$ \\
\hline \multirow[t]{2}{*}{ Male } & 0.036 & 0.034 & 0.033 & 0.033 & $0.050^{\star \star}$ \\
\hline & $(0.000)^{\star \star}$ & $(0.000)^{\star \star}$ & $(0.004)^{\star \star}$ & $(0.004)^{\star \star}$ & $(0.025)$ \\
\hline \multirow[t]{2}{*}{ Age } & 0.002 & 0.002 & 0.002 & 0.002 & 0.008 \\
\hline & $(0.410)$ & $(0.422)$ & $(0.571)$ & $(0.570)$ & $(0.008)$ \\
\hline \multirow[t]{2}{*}{ Black } & -0.010 & -0.010 & -0.029 & -0.029 & \\
\hline & $(0.329)$ & $(0.341)$ & $(0.045)^{*}$ & $(0.042)^{*}$ & \\
\hline \multirow[t]{2}{*}{ Hispanic } & -0.030 & -0.030 & -0.027 & -0.027 & \\
\hline & $(0.003)^{\star \star}$ & $(0.003)^{\star \star}$ & $(0.179)$ & $(0.180)$ & \\
\hline \multirow[t]{2}{*}{ Other Race } & -0.040 & -0.039 & -0.081 & -0.082 & \\
\hline & $(0.007)^{\star \star}$ & $(0.007)^{\star \star}$ & $(0.000)^{\star \star}$ & $(0.000)^{\star \star}$ & \\
\hline \multirow[t]{2}{*}{ General Health Status } & -0.013 & -0.013 & -0.015 & -0.015 & -0.015 \\
\hline & $(0.000)^{\star \star}$ & $(0.000)^{\star \star}$ & $(0.023)^{\star}$ & $(0.024)^{*}$ & $(0.013)$ \\
\hline \multirow[t]{2}{*}{ Birth Order } & 0.004 & 0.004 & 0.007 & 0.007 & 0.015 \\
\hline & $(0.269)$ & $(0.271)$ & $(0.283)$ & $(0.278)$ & $(0.015)$ \\
\hline \multirow[t]{2}{*}{ Parental Age } & -0.000 & -0.000 & -0.001 & -0.001 & \\
\hline & $(0.444)$ & $(0.440)$ & $(0.258)$ & $(0.252)$ & \\
\hline \multirow[t]{2}{*}{ Maternal Education } & -0.006 & -0.006 & -0.002 & -0.002 & \\
\hline & $(0.001)^{\star \star}$ & $(0.001)^{\star \star}$ & $(0.426)$ & $(0.439)$ & \\
\hline \multirow[t]{2}{*}{ Family Income } & -0.000 & -0.000 & -0.000 & -0.000 & \\
\hline & $(0.267)$ & $(0.266)$ & $(0.370)$ & $(0.372)$ & \\
\hline \multirow[t]{2}{*}{ Married Household } & -0.026 & -0.026 & -0.020 & -0.020 & \\
\hline & $(0.000)^{\star \star}$ & $(0.000)^{\star \star}$ & $(0.158)$ & $(0.153)$ & \\
\hline \multirow[t]{2}{*}{ Number Siblings } & -0.004 & -0.003 & -0.003 & -0.003 & \\
\hline & $(0.292)$ & $(0.302)$ & $(0.603)$ & $(0.601)$ & \\
\hline \multirow[t]{2}{*}{ Male X Adhd } & & 0.031 & & & \\
\hline & & $(0.136)$ & & & \\
\hline \multirow[t]{2}{*}{ Sibling ADHD } & & & & -0.014 & \\
\hline & & & & $(0.417)$ & \\
\hline Observations & 12172 & 12172 & 2668 & 2668 & 2668 \\
\hline R-squared & 0.034 & 0.035 & 0.032 & 0.032 & 0.03 \\
\hline Number of Families & & & & & 1526 \\
\hline
\end{tabular}

Notes: ** Statistically significant at $99 \%$ level * statistically significant at $95 \%$ level, +

statistically significant at the $90 \%$ level. All results use robust standard errors clustered at the school or family level. Results for the constant are omitted. Results in Table 2 in the text are calculated by multiplying the ADHD scale coefficient in Table 2A by the standard deviation of the scale (8.89 in Add Health and 3.81 in NLS-Y) 
Table 4A

Association between ADHD Symptoms and Grade Point Average

\begin{tabular}{|l|c|c|c|c|c|}
\hline Outcome & GPA & GPA & GPA & GPA & GPA \\
\hline Sample & Full & Full & Family & Family & Family \\
\hline Fixed Effects? & None & None & None & None & Family \\
\hline ADHD Status & -0.248 & -0.296 & -0.188 & -0.185 & -0.148 \\
\hline & $(0.000)^{\star \star}$ & $(0.000)^{\star \star}$ & $(0.000)^{\star \star}$ & $(0.000)^{\star \star}$ & $(0.099)$ \\
\hline Male & -0.232 & -0.239 & -0.249 & -0.248 & -0.265 \\
\hline & $(0.000)^{\star \star}$ & $(0.000)^{\star \star}$ & $(0.000)^{\star \star}$ & $(0.000)^{\star \star}$ & $(0.057)^{\star \star \star}$ \\
\hline Age & -0.037 & -0.037 & -0.035 & -0.035 & -0.003 \\
\hline & $(0.000)^{\star \star}$ & $(0.000)^{\star \star}$ & $(0.000)^{\star \star}$ & $(0.000)^{\star \star}$ & $(0.021)$ \\
\hline Black & -0.241 & -0.240 & -0.238 & -0.240 & \\
\hline & $(0.000)^{\star \star}$ & $(0.000)^{\star \star}$ & $(0.000)^{\star \star}$ & $(0.000)^{\star \star}$ & \\
\hline Hispanic & -0.191 & -0.191 & -0.225 & -0.225 & \\
\hline & $(0.000)^{\star \star}$ & $(0.000)^{\star \star}$ & $(0.000)^{\star \star}$ & $(0.000)^{\star \star}$ & \\
\hline Other Race & 0.120 & 0.121 & 0.126 & 0.123 & \\
\hline & $(0.068)+$ & $(0.067)+$ & $(0.036)^{\star}$ & $(0.042)^{\star}$ & \\
\hline General Health Status & 0.146 & 0.146 & 0.155 & 0.155 & 0.123 \\
\hline & $(0.000)^{\star \star}$ & $(0.000)^{\star \star}$ & $(0.000)^{\star \star}$ & $(0.000)^{\star \star}$ & $(0.036)^{\star \star \star}$ \\
\hline Birth Order & -0.038 & -0.038 & -0.040 & -0.039 & 0.010 \\
\hline & $(0.002)^{\star \star}$ & $(0.002)^{\star \star}$ & $(0.019)^{\star}$ & $(0.020)^{\star}$ & $(0.035)$ \\
\hline Parental Age & 0.005 & 0.005 & 0.002 & 0.002 & \\
\hline & $(0.003)^{\star \star}$ & $(0.003)^{\star \star}$ & $(0.423)$ & $(0.437)$ & \\
\hline Maternal Education & 0.043 & 0.043 & 0.042 & 0.042 & \\
\hline & $(0.000)^{\star \star}$ & $(0.000)^{\star \star}$ & $(0.000)^{\star \star}$ & $(0.000)^{\star \star}$ & \\
\hline Family Income & 0.001 & 0.001 & 0.001 & 0.001 & \\
\hline & $(0.000)^{\star \star}$ & $(0.000)^{\star \star}$ & $(0.001)^{\star \star}$ & $(0.001)^{\star \star}$ & \\
\hline Married Household & 0.085 & 0.085 & 0.080 & 0.079 & \\
\hline & $(0.000)^{\star \star}$ & $(0.000)^{\star \star}$ & $(0.017)^{\star}$ & $(0.019)^{\star}$ & \\
\hline Number Siblings & 0.028 & 0.028 & 0.034 & 0.034 & \\
\hline & $(0.004)^{\star \star}$ & $(0.004)^{\star \star}$ & $(0.018)^{\star}$ & $(0.018)^{\star}$ & \\
\hline Male X Adhd & & 0.081 & & & \\
\hline Sibling ADHD & $(0.059)+$ & & & \\
\hline Constant & 0.139 & 0.139 & 0.135 & 0.135 & 0.07 \\
\hline Observations & & & & & 1657 \\
\hline R-squared & & & & $(0.225)$ & \\
\hline Number of Families & & & & & \\
\hline
\end{tabular}

Notes: ** Statistically significant at $99 \%$ level * statistically significant at $95 \%$ level, + statistically significant at the $90 \%$ level. All results use robust standard errors clustered at the school or family level. 
Table 5A

Association between ADHD Symptoms and Suspensions

\begin{tabular}{|c|c|c|c|c|c|}
\hline Outcome & Suspended & Suspended & Suspended & Suspended & Suspended \\
\hline Sample & Full & Full & Family & Family & Family \\
\hline Fixed Effects? & None & None & None & None & Family \\
\hline \multirow[t]{2}{*}{ ADHD Status } & 0.155 & 0.147 & 0.174 & 0.171 & $0.125^{\star \star}$ \\
\hline & $(0.000)^{\star \star}$ & $(0.000)^{\star \star}$ & $(0.000)^{\star \star}$ & $(0.000)^{\star \star}$ & $(0.058)$ \\
\hline \multirow[t]{2}{*}{ Male } & 0.143 & 0.142 & 0.135 & 0.134 & $0.132^{\star \star \star}$ \\
\hline & $(0.000)^{\star \star}$ & $(0.000)^{\star \star}$ & $(0.000)^{\star \star}$ & $(0.000)^{\star \star}$ & $(0.036)$ \\
\hline \multirow[t]{2}{*}{ Age } & 0.026 & 0.026 & 0.020 & 0.020 & $0.024^{\star \star}$ \\
\hline & $(0.000)^{\star \star}$ & $(0.000)^{\star \star}$ & $(0.000)^{\star \star}$ & $(0.000)^{\star \star}$ & $(0.012)$ \\
\hline \multirow[t]{2}{*}{ Black } & 0.157 & 0.157 & 0.186 & 0.188 & \\
\hline & $(0.000)^{\star \star}$ & $(0.000)^{\star \star}$ & $(0.000)^{\star \star}$ & $(0.000)^{\star \star}$ & \\
\hline \multirow[t]{2}{*}{ Hispanic } & -0.001 & -0.001 & 0.031 & 0.030 & \\
\hline & $(0.967)$ & $(0.970)$ & $(0.286)$ & $(0.289)$ & \\
\hline \multirow[t]{2}{*}{ Other Race } & -0.050 & -0.050 & -0.047 & -0.043 & \\
\hline & $(0.006)^{\star \star}$ & $(0.006)^{\star \star}$ & $(0.073)+$ & $(0.102)$ & \\
\hline \multirow[t]{2}{*}{ General Health Status } & -0.040 & -0.040 & -0.039 & -0.039 & -0.021 \\
\hline & $(0.000)^{\star \star}$ & $(0.000)^{\star \star}$ & $(0.000)^{\star \star}$ & $(0.000)^{\star \star}$ & $(0.020)$ \\
\hline \multirow[t]{2}{*}{ Birth Order } & 0.011 & 0.011 & 0.005 & 0.005 & 0.004 \\
\hline & $(0.031)^{\star}$ & $(0.031)^{\star}$ & $(0.607)$ & $(0.635)$ & $(0.021)$ \\
\hline \multirow[t]{2}{*}{ Parental Age } & -0.003 & -0.003 & -0.000 & -0.000 & \\
\hline & $(0.000)^{\star \star}$ & $(0.000)^{\star \star}$ & $(0.875)$ & $(0.898)$ & \\
\hline \multirow[t]{2}{*}{ Maternal Education } & -0.018 & -0.018 & -0.023 & -0.023 & \\
\hline & $(0.000)^{\star \star}$ & $(0.000)^{\star \star}$ & $(0.000)^{\star \star}$ & $(0.000)^{\star \star}$ & \\
\hline \multirow[t]{2}{*}{ Family Income } & -0.000 & -0.000 & -0.001 & -0.001 & \\
\hline & $(0.000)^{\star \star}$ & $(0.000)^{\star \star}$ & $(0.001)^{\star \star}$ & $(0.001)^{\star \star}$ & \\
\hline \multirow[t]{2}{*}{ Married Household } & -0.068 & -0.068 & -0.065 & -0.064 & \\
\hline & $(0.000)^{\star \star}$ & $(0.000)^{\star \star}$ & $(0.001)^{\star \star}$ & $(0.001)^{\star \star}$ & \\
\hline \multirow[t]{2}{*}{ Number Siblings } & -0.007 & -0.007 & -0.012 & -0.012 & \\
\hline & $(0.059)+$ & $(0.059)+$ & $(0.156)$ & $(0.159)$ & \\
\hline \multirow[t]{2}{*}{ Male X Adhd } & & 0.014 & & & \\
\hline & & $(0.696)$ & & & \\
\hline \multirow[t]{2}{*}{ Sibling ADHD } & & & & 0.066 & \\
\hline & & & & $(0.019)^{\star}$ & \\
\hline \multirow[t]{2}{*}{ Constant } & 0.255 & 0.256 & 0.344 & 0.340 & -0.167 \\
\hline & $(0.001)^{\star \star}$ & $(0.001)^{\star \star}$ & $(0.002)^{\star \star}$ & $(0.002)^{\star \star}$ & $(0.243)$ \\
\hline Observations & 13565 & 13565 & 2902 & 2902 & 2902 \\
\hline R-squared & 0.110 & 0.110 & 0.120 & 0.122 & 0.05 \\
\hline Number of Families & & & & & 1645 \\
\hline
\end{tabular}

Notes: ${ }^{* *}$ Statistically significant at $99 \%$ level * statistically significant at $95 \%$ level, +

statistically significant at the $90 \%$ level. All results use robust standard errors clustered at the school or family level. 
Table 6A

Association between ADHD Symptoms and Expulsion

\begin{tabular}{|l|c|c|c|c|c|}
\hline Outcome & Expelled & Expelled & Expelled & Expelled & Expelled \\
\hline Sample & Full & Full & Family & Family & Family \\
\hline Fixed Effects? & None & None & None & None & Family \\
\hline ADHD Status & 0.026 & 0.029 & 0.014 & 0.013 & 0.010 \\
\hline & $(0.001)^{\star \star}$ & $(0.004)^{\star \star}$ & $(0.293)$ & $(0.302)$ & $(0.026)$ \\
\hline Male & 0.029 & 0.030 & 0.019 & 0.019 & 0.017 \\
\hline & $(0.000)^{\star \star}$ & $(0.000)^{\star \star}$ & $(0.003)^{\star \star}$ & $(0.004)^{\star \star}$ & $(0.014)$ \\
\hline Age & 0.006 & 0.006 & 0.004 & 0.004 & 0.005 \\
\hline & $(0.000)^{\star \star}$ & $(0.000)^{\star \star}$ & $(0.029)^{\star}$ & $(0.029)^{\star}$ & $(0.005)$ \\
\hline Black & 0.042 & 0.042 & 0.035 & 0.036 & \\
\hline & $(0.000)^{\star \star}$ & $(0.000)^{\star \star}$ & $(0.000)^{\star \star}$ & $(0.000)^{\star \star}$ & \\
\hline Hispanic & 0.018 & 0.018 & 0.037 & 0.036 & \\
\hline & $(0.014)^{\star}$ & $(0.014)^{\star}$ & $(0.007)^{\star \star}$ & $(0.007)^{\star \star}$ & \\
\hline Other Race & -0.000 & -0.000 & 0.016 & 0.016 & \\
\hline & $(0.985)$ & $(0.980)$ & $(0.182)$ & $(0.174)$ & \\
\hline General Health Status & -0.008 & -0.008 & -0.004 & -0.004 & -0.002 \\
\hline & $(0.000)^{\star \star}$ & $(0.000)^{\star \star}$ & $(0.321)$ & $(0.323)$ & $(0.010)$ \\
\hline Birth Order & 0.005 & 0.005 & 0.006 & 0.006 & 0.003 \\
\hline & $(0.017)^{\star}$ & $(0.017)^{\star}$ & $(0.095)+$ & $(0.098)+$ & $(0.010)$ \\
\hline Parental Age & -0.001 & -0.001 & -0.000 & -0.000 & \\
\hline & $(0.002)^{\star \star}$ & $(0.002)^{\star \star}$ & $(0.902)$ & $(0.910)$ & \\
\hline Maternal Education & -0.004 & -0.004 & -0.003 & -0.004 & \\
\hline & $(0.000)^{\star \star}$ & $(0.000)^{\star \star}$ & $(0.011)^{\star}$ & $(0.010)^{\star \star}$ & \\
\hline Family Income & -0.000 & -0.000 & 0.000 & 0.000 & \\
\hline & $(0.188)$ & $(0.185)$ & $(0.846)$ & $(0.851)$ & \\
\hline Married Household & -0.017 & -0.017 & -0.015 & -0.015 & \\
\hline & $(0.000)^{\star \star}$ & $(0.000)^{\star \star}$ & $(0.044)^{\star}$ & $(0.045)^{\star}$ & \\
\hline Number Siblings & -0.002 & -0.002 & -0.006 & -0.006 & \\
\hline & $(0.128)$ & $(0.128)$ & $(0.038)^{\star}$ & $(0.038)^{\star}$ & \\
\hline Male X Adhd & & -0.004 & & & \\
\hline Sibling ADHD & & $(0.763)$ & & & \\
\hline Constant & & & & 0.008 & \\
\hline & $0.080)+$ & $(0.081)+$ & $(0.795)$ & $(0.806)$ & $(0.101)$ \\
\hline Observations & 13963 & 13963 & 2995 & 2995 & 2995 \\
\hline Number of Families & 0.031 & 0.031 & 0.023 & 0.023 & 0.00 \\
\hline
\end{tabular}

Notes: ** Statistically significant at $99 \%$ level * statistically significant at $95 \%$ level, + statistically significant at the $90 \%$ level. All results use robust standard errors clustered at the school or family level. 
Table 7A

Association between ADHD Symptoms and Years of Schooling

\begin{tabular}{|c|c|c|c|c|c|}
\hline Outcome & $\begin{array}{l}\text { Years of } \\
\text { Schooling }\end{array}$ & $\begin{array}{l}\text { Years of } \\
\text { Schooling }\end{array}$ & $\begin{array}{l}\text { Years of } \\
\text { Schooling }\end{array}$ & $\begin{array}{l}\text { Years of } \\
\text { Schooling }\end{array}$ & $\begin{array}{l}\text { Years of } \\
\text { Schooling }\end{array}$ \\
\hline Sample & Full & Full & Family & Family & Family \\
\hline Fixed Effects? & None & None & None & None & Family \\
\hline \multirow[t]{2}{*}{ ADHD Status } & -0.515 & -0.559 & -0.223 & -0.205 & 0.205 \\
\hline & $(0.000)^{\star \star}$ & $(0.000)^{\star \star}$ & $(0.100)+$ & $(0.123)$ & $(0.230)$ \\
\hline \multirow[t]{2}{*}{ Male } & -0.395 & -0.401 & -0.359 & -0.350 & -0.491 \\
\hline & $(0.000)^{\star \star}$ & $(0.000)^{\star \star}$ & $(0.000)^{\star \star}$ & $(0.000)^{\star *}$ & $(0.134)^{\star \star \star}$ \\
\hline \multirow[t]{2}{*}{ Age } & 0.224 & 0.224 & 0.226 & 0.226 & 0.246 \\
\hline & $(0.000)^{\star \star}$ & $(0.000)^{\star \star}$ & $(0.000)^{\star \star}$ & $(0.000)^{\star \star}$ & $(0.044)^{\star \star \star}$ \\
\hline \multirow[t]{2}{*}{ Black } & -0.144 & -0.143 & -0.258 & -0.269 & \\
\hline & $(0.185)$ & $(0.187)$ & $(0.007)^{\star \star}$ & $(0.005)^{\star \star}$ & \\
\hline \multirow[t]{2}{*}{ Hispanic } & -0.035 & -0.034 & -0.167 & -0.167 & \\
\hline & $(0.604)$ & $(0.608)$ & $(0.166)$ & $(0.165)$ & \\
\hline \multirow[t]{2}{*}{ Other Race } & 0.554 & 0.554 & 0.355 & 0.336 & \\
\hline & $(0.007)^{\star \star}$ & $(0.007)^{\star \star}$ & $(0.026)^{\star}$ & $(0.037)^{\star}$ & \\
\hline \multirow[t]{2}{*}{ General Health Status } & 0.309 & 0.309 & 0.329 & 0.328 & 0.158 \\
\hline & $(0.000)^{\star \star}$ & $(0.000)^{\star \star}$ & $(0.000)^{\star \star}$ & $(0.000)^{\star \star}$ & $(0.070)^{\star \star}$ \\
\hline \multirow[t]{2}{*}{ Birth Order } & -0.089 & -0.089 & -0.089 & -0.087 & -0.061 \\
\hline & $(0.001)^{\star \star}$ & $(0.001)^{\star \star}$ & $(0.023)^{\star}$ & $(0.026)^{\star}$ & $(0.077)$ \\
\hline \multirow[t]{2}{*}{ Parental Age } & 0.031 & 0.031 & 0.030 & 0.029 & \\
\hline & $(0.000)^{\star \star}$ & $(0.000)^{\star \star}$ & $(0.000)^{\star \star}$ & $(0.000)^{* *}$ & \\
\hline \multirow[t]{2}{*}{ Maternal Education } & 0.202 & 0.202 & 0.201 & 0.203 & \\
\hline & $(0.000)^{\star \star}$ & $(0.000)^{\star \star}$ & $(0.000)^{\star \star}$ & $(0.000)^{\star \star}$ & \\
\hline \multirow[t]{2}{*}{ Family Income } & 0.005 & 0.005 & 0.006 & 0.006 & \\
\hline & $(0.000)^{\star \star}$ & $(0.000)^{\star \star}$ & $(0.000)^{\star \star}$ & $(0.000)^{\star *}$ & \\
\hline \multirow[t]{2}{*}{ Married Household } & 0.353 & 0.353 & 0.179 & 0.171 & \\
\hline & $(0.000)^{\star \star}$ & $(0.000)^{\star \star}$ & $(0.036)^{\star}$ & $(0.044)^{\star}$ & \\
\hline \multirow[t]{2}{*}{ Number Siblings } & 0.005 & 0.005 & 0.063 & 0.063 & \\
\hline & $(0.824)$ & $(0.823)$ & $(0.055)+$ & $(0.058)+$ & \\
\hline \multirow[t]{2}{*}{ Male X Adhd } & & 0.074 & & & \\
\hline & & $(0.463)$ & & & \\
\hline \multirow[t]{2}{*}{ Sibling ADHD } & & & & -0.346 & \\
\hline & & & & $(0.002)^{\star \star}$ & \\
\hline \multirow[t]{2}{*}{ Constant } & 4.106 & 4.110 & 4.020 & 4.047 & 8.644 \\
\hline & $(0.000)^{\star \star}$ & $(0.000)^{\star \star}$ & $(0.000)^{\star \star}$ & $(0.000)^{\star *}$ & $(0.890)^{\star \star \star}$ \\
\hline Observations & 14068 & 14068 & 3019 & 3019 & 3019 \\
\hline R-squared & 0.202 & 0.202 & 0.195 & 0.198 & 0.10 \\
\hline Number of Families & & & & & 1681 \\
\hline
\end{tabular}

Notes: ** Statistically significant at 99\% level * statistically significant at $95 \%$ level, + statistically significant at the $90 \%$ level. All results use robust standard errors clustered at the school or family level. 
Table 8A

Association between ADHD Symptoms and Dropping Out of High School

\begin{tabular}{|c|c|c|c|c|c|}
\hline Outcome & Dropout & Dropout & Dropout & Dropout & Dropout \\
\hline Sample & Full & Full & Family & Family & Family \\
\hline Fixed Effects? & None & None & None & None & Family \\
\hline \multirow[t]{2}{*}{ ADHD Status } & 0.096 & 0.086 & 0.066 & 0.064 & -0.013 \\
\hline & $(0.000)^{\star \star}$ & $(0.000)^{\star \star}$ & $(0.008)^{\star \star}$ & $(0.010)^{\star}$ & $(0.043)$ \\
\hline \multirow[t]{2}{*}{ Male } & 0.038 & 0.036 & 0.040 & 0.039 & 0.055 \\
\hline & $(0.000)^{\star \star}$ & $(0.000)^{\star \star}$ & $(0.001)^{\star \star}$ & $(0.001)^{\star \star}$ & $(0.025)^{\star \star}$ \\
\hline \multirow[t]{2}{*}{ Age } & -0.010 & -0.010 & -0.011 & -0.011 & -0.014 \\
\hline & $(0.000)^{\star \star}$ & $(0.000)^{\star \star}$ & $(0.006)^{\star \star}$ & $(0.006)^{\star \star}$ & $(0.009)$ \\
\hline \multirow[t]{2}{*}{ Black } & -0.007 & -0.007 & -0.003 & -0.002 & \\
\hline & $(0.481)$ & $(0.491)$ & $(0.848)$ & $(0.914)$ & \\
\hline \multirow[t]{2}{*}{ Hispanic } & -0.001 & -0.001 & 0.040 & 0.040 & \\
\hline & $(0.890)$ & $(0.897)$ & $(0.093)+$ & $(0.093)+$ & \\
\hline \multirow[t]{2}{*}{ Other Race } & -0.046 & -0.046 & -0.043 & -0.040 & \\
\hline & $(0.000)^{\star \star}$ & $(0.000)^{\star \star}$ & $(0.018)^{\star}$ & $(0.026)^{\star}$ & \\
\hline \multirow[t]{2}{*}{ General Health Status } & -0.033 & -0.033 & -0.033 & -0.033 & -0.021 \\
\hline & $(0.000)^{\star \star}$ & $(0.000)^{\star \star}$ & $(0.000)^{\star \star}$ & $(0.000)^{\star \star}$ & $(0.014)$ \\
\hline \multirow[t]{2}{*}{ Birth Order } & 0.002 & 0.002 & -0.004 & -0.004 & -0.009 \\
\hline & $(0.578)$ & $(0.576)$ & $(0.612)$ & $(0.583)$ & $(0.017)$ \\
\hline \multirow[t]{2}{*}{ Parental Age } & -0.002 & -0.002 & -0.002 & -0.002 & \\
\hline & $(0.000)^{\star \star}$ & $(0.000)^{\star \star}$ & $(0.091)+$ & $(0.098)+$ & \\
\hline \multirow[t]{2}{*}{ Maternal Education } & -0.018 & -0.018 & -0.017 & -0.017 & \\
\hline & $(0.000)^{\star \star}$ & $(0.000)^{\star \star}$ & $(0.000)^{\star \star}$ & $(0.000)^{\star \star}$ & \\
\hline \multirow[t]{2}{*}{ Family Income } & -0.000 & -0.000 & -0.001 & -0.001 & \\
\hline & $(0.000)^{\star \star}$ & $(0.000)^{\star \star}$ & $(0.003)^{\star \star}$ & $(0.003)^{\star \star}$ & \\
\hline \multirow[t]{2}{*}{ Married Household } & -0.047 & -0.047 & -0.044 & -0.043 & \\
\hline & $(0.000)^{\star \star}$ & $(0.000)^{\star \star}$ & $(0.004)^{\star \star}$ & $(0.005)^{\star \star}$ & \\
\hline \multirow[t]{2}{*}{ Number Siblings } & 0.005 & 0.005 & -0.000 & -0.000 & \\
\hline & $(0.196)$ & $(0.196)$ & $(0.975)$ & $(0.989)$ & \\
\hline \multirow[t]{2}{*}{ Male X Adhd } & & 0.016 & & & \\
\hline & & $(0.506)$ & & & \\
\hline \multirow[t]{2}{*}{ Sibling ADHD } & & & & 0.045 & \\
\hline & & & & $(0.039)^{*}$ & \\
\hline \multirow[t]{2}{*}{ Constant } & 0.766 & 0.766 & 0.780 & 0.778 & 0.431 \\
\hline & $(0.000)^{\star \star}$ & $(0.000)^{\star \star}$ & $(0.000)^{\star \star}$ & $(0.000)^{\star \star}$ & $(0.179)^{\star \star}$ \\
\hline Observations & 13887 & 13887 & 2974 & 2974 & 2974 \\
\hline R-squared & 0.065 & 0.065 & 0.060 & 0.062 & 0.02 \\
\hline Number of Families & & & & & 1675 \\
\hline
\end{tabular}

Notes: ** Statistically significant at 99\% level * statistically significant at $95 \%$ level, +

statistically significant at the $90 \%$ level. All results use robust standard errors clustered at the school or family level. 
Table 9A

Association between ADHD Symptoms and College Enrollment

\begin{tabular}{|c|c|c|c|c|c|}
\hline Outcome & College & College & College & College & College \\
\hline Sample & Full & Full & Family & Family & Family \\
\hline Fixed Effects? & None & None & None & None & Family \\
\hline \multirow[t]{2}{*}{ ADHD Status } & -0.081 & -0.083 & -0.061 & -0.055 & 0.003 \\
\hline & $(0.000)^{\star \star}$ & $(0.001)^{\star \star}$ & $(0.073)+$ & $(0.098)+$ & $(0.070)$ \\
\hline \multirow[t]{2}{*}{ Male } & -0.074 & -0.074 & -0.055 & -0.055 & -0.094 \\
\hline & $(0.000)^{\star \star}$ & $(0.000)^{\star \star}$ & $(0.004)^{\star \star}$ & $(0.005)^{\star \star}$ & $(0.042)^{\star \star}$ \\
\hline \multirow[t]{2}{*}{ Age } & -0.010 & -0.010 & -0.003 & -0.003 & 0.006 \\
\hline & $(0.014)^{*}$ & $(0.014)^{\star}$ & $(0.571)$ & $(0.569)$ & $(0.014)$ \\
\hline \multirow[t]{2}{*}{ Black } & -0.021 & -0.021 & -0.037 & -0.040 & \\
\hline & $(0.277)$ & $(0.276)$ & $(0.153)$ & $(0.131)$ & \\
\hline \multirow[t]{2}{*}{ Hispanic } & 0.039 & 0.039 & 0.058 & 0.059 & \\
\hline & $(0.042)^{*}$ & $(0.042)^{\star}$ & $(0.077)^{+}$ & $(0.075)+$ & \\
\hline \multirow[t]{2}{*}{ Other Race } & 0.091 & 0.091 & 0.047 & 0.043 & \\
\hline & $(0.010)^{*}$ & $(0.010)^{\star \star}$ & $(0.212)$ & $(0.261)$ & \\
\hline \multirow[t]{2}{*}{ General Health Status } & 0.059 & 0.059 & 0.056 & 0.056 & 0.012 \\
\hline & $(0.000)^{\star *}$ & $(0.000)^{\star \star}$ & $(0.000)^{\star \star}$ & $(0.000)^{\star \star}$ & $(0.023)$ \\
\hline \multirow[t]{2}{*}{ Birth Order } & -0.018 & -0.018 & -0.023 & -0.022 & -0.006 \\
\hline & $(0.001)^{\star \star}$ & $(0.001)^{\star \star}$ & $(0.047)^{\star}$ & $(0.054)+$ & $(0.023)$ \\
\hline \multirow[t]{2}{*}{ Parental Age } & 0.006 & 0.006 & 0.006 & 0.006 & \\
\hline & $(0.000)^{\star \star}$ & $(0.000)^{\star \star}$ & $(0.004)^{\star \star}$ & $(0.005)^{\star \star}$ & \\
\hline \multirow{2}{*}{ Maternal Education } & 0.043 & 0.043 & 0.045 & 0.046 & \\
\hline & $(0.000)^{\star *}$ & $(0.000)^{\star \star}$ & $(0.000)^{\star \star}$ & $(0.000)^{\star \star}$ & \\
\hline \multirow[t]{2}{*}{ Family Income } & 0.001 & 0.001 & 0.001 & 0.001 & \\
\hline & $(0.000)^{\star *}$ & $(0.000)^{\star \star}$ & $(0.000)^{\star \star}$ & $(0.000)^{\star \star}$ & \\
\hline \multirow[t]{2}{*}{ Married Household } & 0.051 & 0.051 & 0.029 & 0.027 & \\
\hline & $(0.000)^{\star \star}$ & $(0.000)^{\star \star}$ & $(0.202)$ & $(0.234)$ & \\
\hline \multirow[t]{2}{*}{ Number Siblings } & 0.001 & 0.001 & 0.015 & 0.014 & \\
\hline & $(0.753)$ & $(0.753)$ & $(0.123)$ & $(0.136)$ & \\
\hline \multirow[t]{2}{*}{ Male X Adhd } & & 0.004 & & & \\
\hline & & $(0.903)$ & & & \\
\hline \multirow[t]{2}{*}{ Sibling ADHD } & & & & -0.092 & \\
\hline & & & & $(0.004)^{\star *}$ & \\
\hline \multirow[t]{2}{*}{ Constant } & -0.221 & -0.220 & -0.380 & -0.377 & 0.541 \\
\hline & $(0.059)+$ & $(0.058)+$ & $(0.005)^{\star \star}$ & $(0.005)^{\star \star}$ & $(0.287)^{*}$ \\
\hline Observations & 12511 & 12511 & 2662 & 2662 & 2662 \\
\hline$R$-squared & 0.106 & 0.106 & 0.095 & 0.098 & 0.01 \\
\hline Number of Families & & & & & 1553 \\
\hline
\end{tabular}

Notes: ** Statistically significant at $99 \%$ level * statistically significant at $95 \%$ level, + statistically significant at the $90 \%$ level. All results use robust standard errors clustered at the school or family level. 
Table 10A

Comparison Across Family Spillover Specifications

Basic Model (from Table 4), Extended Model, and Random Effects Model

\begin{tabular}{|l|c|c|c|c|c|c|c|c|c|}
\hline Outcome & $\begin{array}{c}\text { Years of } \\
\text { Schooling }\end{array}$ & $\begin{array}{c}\text { Years of } \\
\text { Schooling }\end{array}$ & $\begin{array}{c}\text { Years of } \\
\text { Schooling }\end{array}$ & Drop Out & Drop Out & Drop Out & College & College & College \\
\hline Sample & Sibling & Sibling & Sibling & Sibling & Sibling & Sibling & Sibling & Sibling & Sibling \\
\hline Specification & Basic & Extended & RE & Basic & Extended & RE & Basic & Extended & RE \\
\hline ADHD Status & -0.205 & -0.149 & -0.188 & 0.064 & 0.055 & 0.062 & -0.055 & -0.047 & -0.053 \\
\hline & $(0.123)$ & $(0.239)$ & $(0.155)$ & $(0.010)^{\star}$ & $(0.022)^{\star}$ & $(0.012)^{\star}$ & $(0.098)^{+}$ & $(0.137)$ & $(0.120)$ \\
\hline Sibling ADHD & -0.346 & -0.309 & -0.369 & 0.045 & 0.037 & 0.056 & -0.092 & -0.080 & -0.076 \\
\hline & $(0.002)^{\star \star}$ & $(0.004)^{\star *}$ & $(0.002)^{\star *}$ & $(0.039)^{\star}$ & $(0.078)^{+}$ & $(0.013)^{\star}$ & $(0.004)^{\star *}$ & $(0.011)^{\star}$ & $(0.020)^{\star}$ \\
\hline Observations & 3019 & 3019 & 3019 & 2974 & 2974 & 2974 & 2662 & 2662 & 2662 \\
\hline R-squared & 0.198 & 0.273 & & 0.062 & 0.108 & & 0.098 & 0.151 & \\
\hline
\end{tabular}

\begin{tabular}{|l|c|c|c|c|c|c|c|c|c|}
\hline Outcome & GPA & GPA & GPA & Expelled & Expelled & Expelled & Suspended & Suspended & Suspended \\
\hline Sample & Sibling & Sibling & Sibling & Sibling & Sibling & Sibling & Sibling & Sibling & Sibling \\
\hline Specification & Basic & Extended & RE & Basic & Extended & RE & Basic & Extended & RE \\
\hline ADHD Status & -0.185 & -0.172 & -0.174 & 0.013 & 0.011 & 0.011 & 0.171 & 0.163 & 0.161 \\
\hline & $(0.000)^{\star \star}$ & $(0.001)^{\star \star}$ & $(0.001)^{\star \star}$ & $(0.302)$ & $(0.400)$ & $(0.417)$ & $(0.000)^{\star \star}$ & $(0.000)^{\star \star}$ & $(0.000)^{\star \star}$ \\
\hline Sibling ADHD & -0.057 & -0.048 & -0.044 & 0.008 & 0.006 & 0.005 & 0.066 & 0.057 & 0.053 \\
\hline & $(0.225)$ & $(0.298)$ & $(0.359)$ & $(0.472)$ & $(0.572)$ & $(0.630)$ & $(0.019)^{\star}$ & $(0.032)^{\star}$ & $(0.056)+$ \\
\hline Observations & 2944 & 2944 & 2944 & 2995 & 2995 & 2995 & 2902 & 2902 & 2902 \\
\hline R-squared & 0.135 & 0.184 & & 0.023 & 0.037 & & 0.122 & 0.151 & \\
\hline
\end{tabular}

\begin{tabular}{|l|c|c|c|c|c|c|}
\hline Outcome & Repeat Grade & Repeat Grade & Repeat Grade & Special Ed & Special Ed & Special Ed \\
\hline Sample & Sibling & Sibling & Sibling & Sibling & Sibling & Sibling \\
\hline Specification & Basic & Extended & RE & Basic & Extended & RE \\
\hline ADHD Status & 0.050 & 0.048 & 0.051 & 0.060 & 0.057 & 0.065 \\
\hline & $(0.009)^{\star \star}$ & $(0.014)^{\star}$ & $(0.008)^{\star \star}$ & $(0.014)^{\star}$ & $(0.019)^{\star}$ & $(0.009)^{\star \star}$ \\
\hline Sibling ADHD & -0.013 & -0.017 & -0.010 & -0.008 & -0.010 & -0.006 \\
\hline & $(0.308)$ & $(0.192)$ & $(0.450)$ & $(0.654)$ & $(0.578)$ & $(0.761)$ \\
\hline Observations & 3015 & 3015 & 3015 & 2668 & 2668 & 2668 \\
\hline R-squared & 0.048 & 0.062 & & 0.024 & 0.048 & \\
\hline
\end{tabular}

Notes: ${ }^{* *}$ Statistically significant at $99 \%$ level * statistically significant at $95 \%$ level, +

statistically significant at the $90 \%$ level. All results use robust standard errors clustered at the school or family level. Basic models include all covariates listed in Table 9A (column 1).

Extended models include the covariates in the Basic models and the community unemployment rate, the community proportion in poverty, maternal smoking status, father's education level, parent's general health status, parent's age, reports of parents' alcoholism status, receipt of AFDC or Food Stamps, and parental reports of education expectations. Random Effects specifications only contain the following covariates: general health status, birth order, sex, and age. 
Variable Definitions

\begin{tabular}{|c|c|c|c|c|}
\hline Variable & Respondent & Grades & Wave & Question \\
\hline $\begin{array}{l}\text { Grade } \\
\text { Repetition }\end{array}$ & Individual & 7th-12th & 1 & $\begin{array}{l}\text { Have you ever repeated a grade or been held back a } \\
\text { grade (if so, which grade)? }\end{array}$ \\
\hline $\begin{array}{l}\text { Special } \\
\text { Education }\end{array}$ & Parent & $\begin{array}{l}\text { Grade in } \\
\text { Wave } 1\end{array}$ & 1 & $\begin{array}{l}\text { During the last } 12 \text { months, did (he/she) receive any type } \\
\text { of special education service }\end{array}$ \\
\hline Suspended & Individual & 7th-12th & 1 & $\begin{array}{l}\text { Have you ever received an out-of-school suspension } \\
\text { from school (what grade)? }\end{array}$ \\
\hline Expelled & Individual & 7th-12th & 1 & $\begin{array}{l}\text { Have you ever been expelled from school (which } \\
\text { grade)? }\end{array}$ \\
\hline GPA & Individual & $\begin{array}{l}\text { Grade in } \\
\text { Wave } 1\end{array}$ & 1 & $\begin{array}{l}\text { Average of most recent grades received from courses } \\
\text { taken in English, Math, Science, History }\end{array}$ \\
\hline $\begin{array}{l}\text { Years of } \\
\text { Schooling }\end{array}$ & Individual & & 3 & $\begin{array}{l}\text { What is the highest grade or year of regular school you } \\
\text { have completed? }\end{array}$ \\
\hline Drop Out & Individual & & 3 & If highest grade completed $<12$ and not still in school \\
\hline College & Individual & & 3 & If highest grade completed $>12$ \\
\hline
\end{tabular}

$\wedge$ In Wave 1 , the children were between the ages of 13 to 22 . In Wave 3 , the respondents were between the ages of 18 and 28. 
Retrospective Add Health Variables for ADHD

Inattentive Variables

H3RA1-You failed to pay close attention to details or made careless mistakes in your work.

H3RA3-You had difficulty sustaining attention in tasks or fun activities.

H3RA5-You didn't listen when spoken to directly.

H3RA7-You didn't follow through on instructions and failed to finish work.

H3RA9-You had difficulty organizing tasks and activities.

H3RA11 - You avoided, disliked, or were reluctant to engage in work requiring sustained mental effort.

H3RA13-You lost things that were necessary for tasks or activities.

H3RA15-You were easily distracted.

H3RA17-You were forgetful.

Hyperactive Variables

H3RA2-You fidgeted with your hands or feet or squirmed in your seat.

H3RA4-You left your seat in the classroom or in other situations when being seated was expected.

H3RA6-You felt restless.

H3RA8-You had difficulty doing fun things quietly.

H3RA10 - You felt "on the go" or "driven by a motor."

H3RA12-You talked too much.

H3RA14-You blurted out answers before the questions had been completed.

H3RA16-You had difficulty awaiting your turn. 